\section{Diffuse muscle pain with quetiapine}

We report the case of a 28 -year-old female out-patient with bipolar disorder, whose symptomatology was well-controlled with lithium carbonate $1200 \mathrm{mg}$ (orally) $(0.9 \mathrm{mEq} / \mathrm{l}$ plasma levels $)$ and risperidone 1-2 mg (orally) daily. The patient had been treated for several years in our department and the course of her illness was wellknown; it showed that only lithium was both effective and well-tolerated (topiramate was not effective and carbamazepine caused a rash) and only in coadministration with low doses of risperidone.

However, the use of risperidone caused a large increase in prolactin levels (above $2000 \mu \mathrm{U} / \mathrm{l}$, with normal values below $500 \mu \mathrm{U} / \mathrm{l}$ ) and amenorrhoea (the rest of the hormonal investigation and brain magnetic resonance imaging were normal). The patient was put on $5 \mathrm{mg}$ olanzapine (orally), but she did not tolerate this agent because it made her feel 'confused' and 'tired'. She was then put on $200 \mathrm{mg}$ quetiapine (orally). Within $24 \mathrm{~h}$ the patient manifested diffuse muscle pains and headache. She reported that her legs were stiff and she had pain in her knee joints. Neurological examination was normal, as were blood and biochemical tests including creatine phosphokinase. Vital signs were normal. No extrapyramidal signs or symptoms (especially akathisia) were present. The pain persisted for 5 more days and the patient demanded that quetiapine be discontinued. The pain disappeared within the first $48 \mathrm{~h}$ of shifting back to risperidone, which was according to the wishes of the patient. Six months passed and the patient is still free from symptoms.

To our knowledge, this is the first report of this kind of adverse effect related to quetiapine. Various other antipsychotics, including haloperidol and olanzapine, are reported to cause muscle pain and rigidity because of rhabdomyolysis, but the current case had no laboratory or clinical findings related to rhabdomyolysis.

\section{Declaration of interest}

K.N.F. has participated in field trials sponsored by Eli Lilley \& Co. and by Astra-Zeneca.

K. N. Fountoulakis, A. lacovides, St. G. Kaprinis, G. St. Kaprinis 53 Chrysostomou Smyrnis Street, 55132 Aretsou Thessaloniki, Greece

\section{Adjunctive fluvoxamine with clozapine}

We read with interest the article by Williams et al (2002). The authors summarise treatment options for patients resistant to clozapine monotherapy. However, in the section on combining antidepressants with clozapine, several issues deserve more attention. The authors disagree with utilisation of adjunctive antidepressants to reduce the cost of clozapine treatment. In our recent study (Lu et al, 2000), addition of $50 \mathrm{mg} /$ day fluvoxamine to low-dose $(100 \mathrm{mg} /$ day $)$ clozapine could raise the mean plasma clozapine level to over $400 \mathrm{ng} / \mathrm{ml}$ to achieve suitable therapeutic ranges. Therefore, concomitant fluvoxamine can reduce clozapine doses and, consequently, costs (Armstrong \& Cozza, 2001).

Interestingly, this pharmacokinetic interaction is more pronounced in patients with high cytochrome P450 1A2 activity and at low clozapine plasma concentrations (Olesen \& Linnet, 2000). This phenomenon could therefore be used to narrow down the wide interindividual variation in blood clozapine concentrations. Several open trials also demonstrated that coadministration of fluvoxamine could augment clozapine efficacy and curtail plasma norclozapine:clozapine ratios (Wetzel et al, 1998; Lu et al, 2000). Norclozapine has been suggested to be more toxic than its parent compound. Although addition of fluvoxamine to low-dose clozapine was well-tolerated in our pilot study (Lu et al, 2000), further studies are warranted to substantiate its safety and efficacy.

\section{Armstrong, S. C. \& Cozza, K. L. (200I)}

Consultation-liaison psychiatry drug-drug interactions update. Psychosomatics, 42, I57-159.

Lu, M. L., Lane, H. Y., Chen, K. P., et al (2000) Fluvoxamine reduces the clozapine dosage needed in refractory schizophrenic patients. Journal of Clinical Psychiatry, 6I, 594-599.

Olesen, O. V. \& Linnet, K. (2000) Fluvoxamineclozapine drug interaction: inhibition in vitro of five cytochrome P450 isoforms involved in clozapine metabolism. Journal of Clinical Psychopharmacology, 20 $35-42$

Wetzel, H., Anghclescu, I., Szegedi, A., et al (1998) Pharmacokinetic interactions of clozapine with selective serotonin reuptake inhibitors: differential effects of fluvoxamine and paroxetine in a prospective study. Journal of Clinical Psychopharmacology, 18, 2-9.

Williams, L., Newton, G., Roberts, K., et al (2002) Clozapine-resistant schizophrenia: a positive approach British Journal of Psychiatry, 18I, 184-187.

\section{Declaration of interest}

None. Funding was received from the $\mathrm{Na}$ tional Science Council and the National Health Research Institutes, Taiwan.

M. L. Lu Department of Psychiatry, Taipei Medical University-Wan Fang Hospital, Taipei, Taiwan

H. Y. Lane Department of Psychiatry, China Medical College Hospital, No. 2, Yuh-Der Road, Taichung 404, Taiwan

\section{Olanzepine-induced tardive dyskinesia}

Tardive dyskinesia is a serious and common motor side-effect of treatment with traditional neuroleptics, with an unknown pathophysiological basis. It affects 20 $30 \%$ of patients on long-term neuroleptic therapy, with elderly patients being at higher risk (American Psychiatric Association, 1994).

Olanzapine is an atypical antipsychotic agent with a reported lack of propensity to cause tardive dyskinesia (Beasley et al, 1999). Recently, it has been suggested that olanzapine can improve tardive dyskinesia in some patients (Littrell et al, 1998; Jaffe \& Simpson, 1999). Other authors, however, have shown that the prolonged use of olanzapine can instead be associated with tardive dyskinesia/dystonia (Ananth \& Kenan, 1999; Dunayevich \& Strakowski, 1999). Here we report the case of a patient who experienced tardive dyskinesia after only few months of treatment with olanzapine.

A 62-year-old housewife with an unremarkable past medical history, sought out-patient treatment in June 2000 for anxiety, insomnia, difficulty thinking and concentrating, and frequent episodes of aggressive behaviour. She was evaluated by neurologists, and was submitted to routine biochemical investigations (unremarkable), a computerised tomography scan (normal), and the Mini-Mental State Examination (24/30). Olanzapine $(10 \mathrm{mg} /$ day) was started and this was the sole medication continued thereafter. The patient soon experienced a subjective improvement. Three to four months later she noticed slight involuntary movements of the tongue and jaw. Despite these symptoms, she continued taking olanzapine until it was eventually stopped 1.5 years later (December 2001).

She was admitted to our hospital in March 2002. On examination, she displayed marked and distressing involuntary 